\title{
Superficial siderosis of the central nervous system associated with intraspinal hemorrhage from ventral thoracic epidural veins and a ventral spinal CSF leak: case report
}

\author{
Keisuke Takai, MD, PhD, ${ }^{1}$ Takashi Komori, MD, PhD, ${ }^{2}$ Manabu Niimura, MD, PhD, ${ }^{1}$ and \\ Makoto Taniguchi, MD, PhD ${ }^{1}$ \\ Departments of ${ }^{1}$ Neurosurgery, and ${ }^{2}$ Laboratory Medicine and Pathology, Tokyo Metropolitan Neurological Hospital, Tokyo, \\ Japan

\begin{abstract}
In most patients with superficial siderosis of the CNS, the exact source of bleeding remains unknown because of a lack of objective surgical data. The authors herein describe the case of a 58-year-old man with superficial siderosis of the CNS. The patient also had spinal CSF leakage due to a spinal dural defect. Repair surgery for the dural defect was performed using posterior laminoplasty with a transdural approach without spinal fixation. During repair surgery, the bleeding source was found to be the epidural vein around the defect. The intraoperative and histological results of the present case suggest that epidural veins exposed to CSF represent a chronic bleeding source in patients with superficial siderosis of the CNS complicated by CSF leakage. Dural repair surgery may result in discontinuation of the CSF leaks, resolution of the epidural CSF collection, and cessation of chronic epidural bleeding.
\end{abstract} \\ https://thejns.org/doi/abs/10.3171/2016.11.SPINE16488
}

KEY WORDS hemosiderosis; bleeding source; epidural venous plexus; spinal surgery; vascular disorders

$\mathrm{S}$ UPERFICIAL siderosis of the CNS is a progressive neurological syndrome that is characterized by chronic subarachnoid bleeding. Chronic bleeding leads to the deposition of hemosiderin on the superficial layer of the cerebellum, eighth cranial nerve, brainstem, and spinal cord, resulting in severe neurological deficits, such as cerebellar ataxia and hearing impairment. The arrest of bleeding with neurosurgery has the beneficial effect of preventing neurological deterioration; however, the exact source of bleeding remains unknown because of a lack of objective surgical data in most patients with siderosis. Here, we present a case of superficial siderosis of the CNS associated with CSF leakage; the bleeding source was verified based on intraoperative and histological findings.

\section{Case Report}

A 58-year-old man presented with a 5-year history of a slowly progressive gait disturbance and hearing impairment. The patient had no previous history of surgery or trauma in the CNS. Neurological examination revealed an ataxic gait and dysarthria in addition to bilateral sen- sorineural deafness. Brain MRI demonstrated superficial siderosis of the CNS (Fig. 1A and B). No brain tumor or vascular lesion was reported. Lumbar puncture showed a low opening pressure $\left(4 \mathrm{~cm} \mathrm{H}_{2} \mathrm{O}\right)$ and hemorrhagic CSF, and the red blood cell count of the CSF ranged between 2800 and 3300/ $\mu$ l. The patient's hemoglobin concentration and coagulation status were within reference ranges with a hemoglobin concentration of $12 \mathrm{~g} / \mathrm{ml}$, prothrombin time of $90 \%$, and activated thromboplastin time of 35 seconds.

Spinal MRI showed the accumulation of epidural CSF ventral to the dural sac in the cervical and thoracic regions (Fig. 1C and D). No intraspinal bleeding was detected. On thin-slice (3-mm) FIESTA MR images, a dural defect was suspected at several spinal levels from C-4 to T-7; however, there was no conclusive evidence to localize the level of the spinal CSF leak. On CT myelography of the cervical, thoracic, and lumbar spine, the site of the dural defect was predicted to be anywhere between C-7 and T-3, because maximal contrast collection was detected in the ventral epidural space and the spinal cord adhered to the ventral side of the dura mater at the level of T-3 (Fig. 1E). 

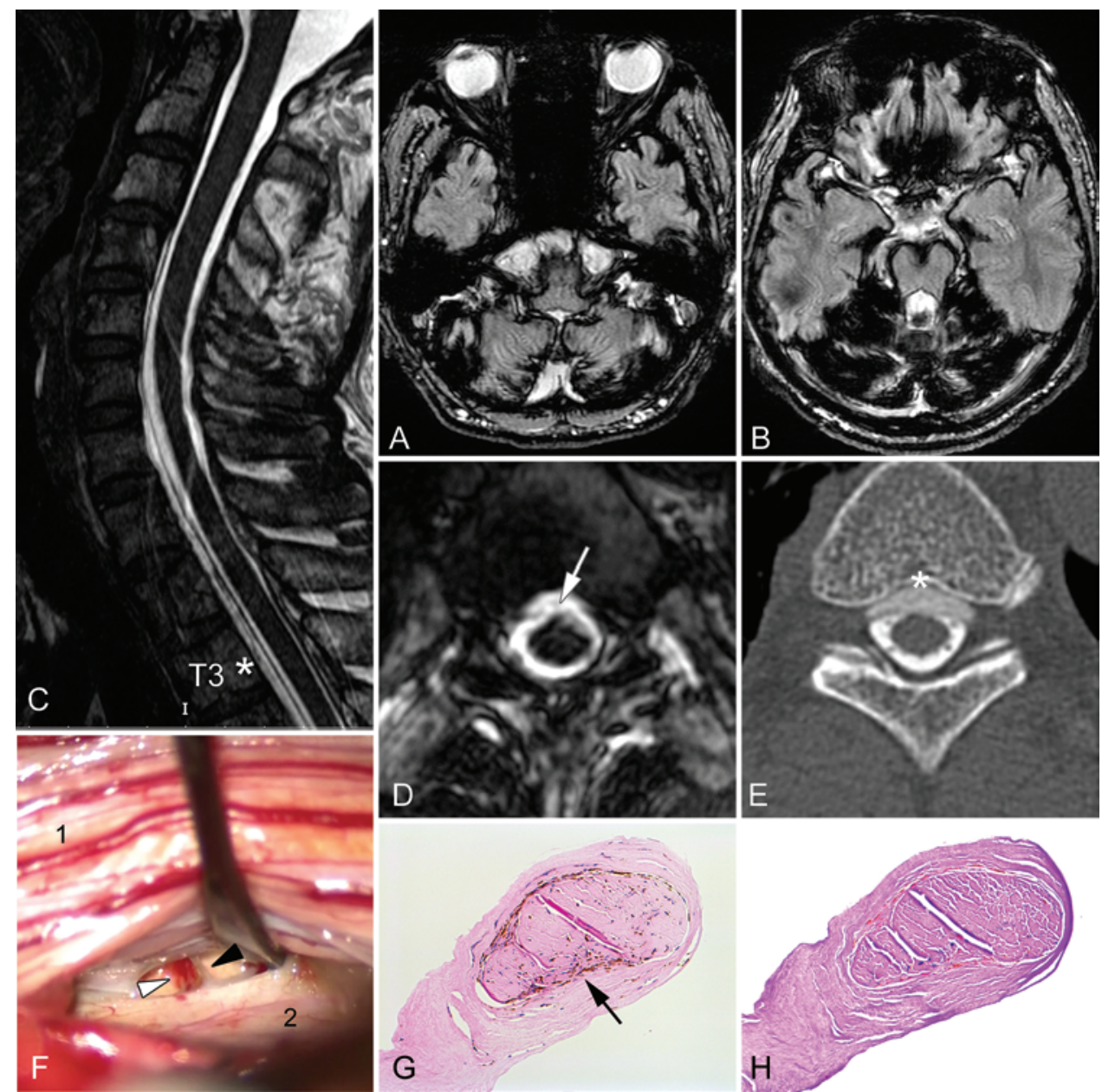

FIG. 1. A and B: Axial gradient echo MR images of the brain. C and D: Sagittal (C) and axial (D) FIESTA MR images of the spine. The asterisk indicates the epidural fluid collection, and the arrow indicates a dural defect. E: Axial CT myelogram. The asterisk indicates the epidural contrast collection. F: Intraoperative image showing a dural defect. 1 denotes the spinal cord; 2 , the dura mater; white arrowhead, a fresh hematoma; and black arrowhead, the epidural tissue. $\mathbf{G}$ and $\mathbf{H}$ : Photomicrographs of epidural tissue. The arrow indicates hemosiderin deposition. $\mathrm{H} \& \mathrm{E}(\mathrm{G})$; Elastica van Gieson $(\mathrm{H})$. Original magnification $\times 200$. Figure is available in color online only.

To identify the bleeding source and repair the suspected dural defect, the patient underwent neurosurgery. The patient was positioned prone, and laminoplasty from C-7 to T-3 and dorsal durotomy were performed. To gain intradural exposure of the ventral dural defect, the lateral attachments of the dentate ligament were cut at the C-7 to T-3 levels on the right side, while the spinal arachnoid remained intact.

During surgery, the dural defect $(5 \times 2 \mathrm{~mm})$ was detected at the level of T-3 ventral to the spinal cord (Fig. 1F). There was also an arachnoid defect at the same site, and the arachnoid stump adhered to the dural defect circumferentially. The spinal subarachnoid space had free communication with the ventral epidural space through the defect. A small fresh hematoma around the epidural tissue was found in the ventral epidural space. To clarify the source of bleeding, ventral epidural tissue was biopsied from an intradural approach. A histological examination of the biopsy sample revealed an organized vessel lacking the elastic lamina, which is compatible with a thrombosed epidural vein. The deposition of hemosiderin along the surface of the residual vessel lumen suggested continuous bleeding from this epidural tissue (Fig. $1 \mathrm{G}$ and $\mathrm{H}$ ). The dural defect was repaired with the autologous fascia of the neck muscle. After dural repair, laminae from C-7 to T-3 were fixed with titanium plates. Spinal fixation was not performed because the spinal facet joints and pedicles remained intact.

The epidural CSF collection resolved and intradural CSF became clear and bloodless postoperatively. The patient's cerebellar ataxia and the hearing impairment partially improved. Preoperatively, the patient communicated with others by means of writing because of ataxic dysarthria, and sensorineural deafness; however, he was able to communicate by speaking and listening postoperatively. The patient became ambulant without a cane 1 month after surgery. The recurrence of bleeding was not reported in the CSF examination performed 7 months after dural repair. The epidural CSF collection resolved, and there was no spinal deformity or instability on MR images 1 year after dural repair (Fig. 2). 

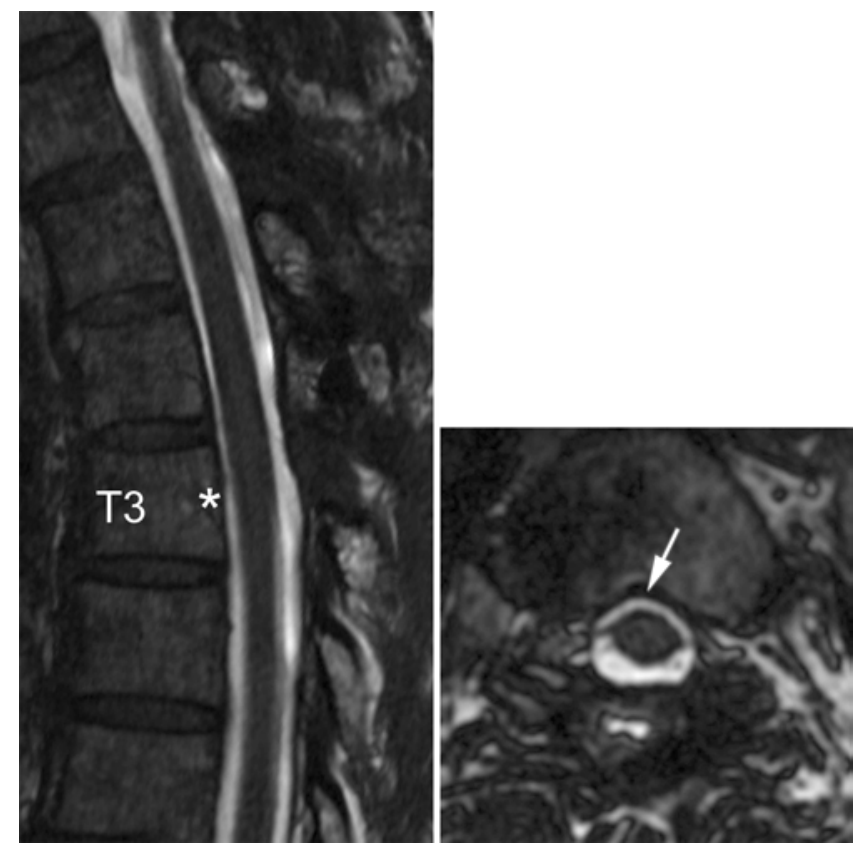

FIG. 2. Postoperative sagittal (left) and axial (right) FIESTA MR images of the spine. The asterisk indicates that there is no epidural fluid collection, and the arrow indicates the lack of a dural defect.

\section{Discussion}

Two theories have been proposed for the source of bleeding in patients with superficial siderosis of the CNS associated with a ventral spinal CSF leak. The first theory is that brain sagging due to intracranial hypotension may lead to bleeding from bridging veins on the surface of the cerebellum, ${ }^{3}$ and the second theory is that the bleeding source may be fragile vessels around the dural defect. ${ }^{2,4,5}$ However, the exact bleeding source remains unknown because of a lack of objective data, even in patients who undergo neurosurgery. ${ }^{1}$ We herein showed, for the first time, based on intraoperative and histological results, that the epidural vein around the defect was the bleeding source of superficial siderosis of the CNS associated with a ventral spinal CSF leak. These results support the second theory. Based on our results, the etiology of superficial siderosis of the CNS associated with CSF leaks may be explained as follows. When CSF leaks through a dural defect, the epidural space enlarges because of shrinking of the dural sac, resulting in an enlarged epidural venous plexus with a fluid-filled collection over a large area in the spinal canal. When a small amount of bleeding occurs from the epidural veins, it may not always stop spontaneously because CSF continues to flow and remove clots over the epidural veins, leading to subarachnoid bleeding through the dural defect. Repair surgery for the dural defect may be performed using posterior laminoplasty with a transdural approach without spinal fixation. Dural repair surgery may lead to the arrest of CSF leaks, resolution of the epidural CSF collection, and cessation of chronic epidural bleeding.

\section{References}

1. Egawa S, Yoshii T, Sakaki K, Inose H, Kato T, Kawabata S, et al: Dural closure for the treatment of superficial siderosis. J Neurosurg Spine 18:388-393, 2013

2. Kumar N: Beyond superficial siderosis: introducing "duropathies." Neurology 78:1992-1999, 2012

3. Schievink WI, Maya MM, Nuño M: Chronic cerebellar hemorrhage in spontaneous intracranial hypotension: association with ventral spinal cerebrospinal fluid leaks: clinical article. J Neurosurg Spine 15:433-440, 2011

4. Schievink WI, Wasserstein P, Maya MM: Intraspinal hemorrhage in spontaneous intracranial hypotension: link to superficial siderosis? Report of 2 cases. J Neurosurg Spine 24:454-456, 2016

5. Yokosuka J, Takai K, Komori T, Taniguchi M: Superficial siderosis: bleeding from the bone marrow after laminectomy for spinal tumor removal. J Neurosurg Spine 21:905-908, 2014

\section{Disclosures}

The authors report no conflict of interest concerning the materials or methods used in this study or the findings specified in this paper.

\section{Author Contributions}

Conception and design: Takai. Acquisition of data: Takai, Niimura. Analysis and interpretation of data: Takai, Komori. Drafting the article: Takai. Critically revising the article: Komori, Taniguchi. Reviewed submitted version of manuscript: all authors. Approved the final version of the manuscript on behalf of all authors: Takai. Study supervision: Takai.

\section{Correspondence}

Keisuke Takai, Department of Neurosurgery, Tokyo Metropolitan Neurological Hospital, 2-6-1, Musashidai, Fuchu, Tokyo 1830042, Japan. email: takai-nsu@umin.ac.jp. 